\title{
Fabricación y caracterización mecánica de un material compuesto de matriz polimérica y carga cerámica
}

\author{
A. ARIAS, R. ZAERA, J. LÓPEZ-PUENTE. C. NAVARRO \\ Dpto. Mecánica de Medios Continuos y Teoría de Estructuras, Universidad Carlos III de Madrid 28911 Leganés, Madrid, España
}

\begin{abstract}
En este trabajo se presenta el proceso de fabricación de un material compuesto de matriz polimérica y carga cerámica, su caracterización mecánica estática y su comportamiento frente a impacto. Como matriz se ha empleado una resina viniléster y como carga cerámica se han empleado partículas de alúmina y carburo de silicio. Estas partículas, con un tamaño medio comprendido entre $1.5 \mathrm{~mm}$ y $4.5 \mathrm{~mm}$, presentan bajo coste y alta disponibilidad. La compactación uniaxial permitió obtener losetas con un elevado porcentaje de partículas cerámicas (hasta el 80\% en peso) y baja porosidad. Se han realizado ensayos de compresión estática sobre el material desarrollado para analizar la influencia de varios factores: fracción volumétrica de partículas, tamaño de partícula, material de las partículas y temperatura. La resistencia a compresión cuasiestática del nuevo material compuesto se incrementa al aumentar la fracción volumétrica de partículas, siendo prácticamente independiente del tamaño de éstas, en el rango granulométrico considerado. El incremento de la temperatura supone una disminución en las propiedades cuasiestáticas. El bajo coste del material desarrollado justifica su aplicación en protecciones que requieran de una eficiencia frente a impacto intermedia entre la correspondiente a placas metálicas y a los paneles cerámica/metal.
\end{abstract}

Palabras clave: Partículas cerámicas, Matriz polimérica, Eficiencia balística, Compresión estática, Ensayo de profundidad de penetración.

\section{Manufacturing and compressive behavior of a polymeric material loaded with ceramic particles}

A polymeric material loaded with ceramic particles was developed and its static and impact behaviors were determined. A vinylester resin, used as matrix, was reinforced with alumina or silicon carbide particles. These particles, with mean sizes between 1.5 and $4.5 \mathrm{~mm}$, are cheap and readily obtainable. The manufacturing process produced tiles with a high percentage of ceramic particles (up to $80 \%$ by weight) and low porosity. The static compressive behavior of the composite was examined to consider various factors: ceramic fraction, particle size, material of particles and temperature. A higher volumetric fraction of particles produces an increase of stiffness and raises the yield stress of the composite; the influence of size of particles is not determinant. High temperatures produces a decrease of the mechanical properties. The efficiency of this low-cost material is halfway between that of the conventional metallic materials and the monolithic ceramic tiles used in lightweight protection.

Keywords: Ceramic particles, Polymeric matrix, Ballistic efficiency, Static compression, Depth of penetration test.

\section{INTRODUCCIÓN}

En el proceso de diseño de protecciones frente a impacto, los requerimientos más importantes son elevada capacidad de detención de la amenaza, baja densidad y reducido coste de los materiales. Se pueden emplear diferentes materiales para satisfacer estos requerimientos. Los materiales metálicos pueden, en general, responder a los requerimientos de protección a un bajo coste, pero presentan el inconveniente de su elevada densidad. El uso de los materiales cerámicos está restringido por su baja tenacidad, dado que se fragmentan fácilmente ante cargas impulsivas. No obstante, estos últimos, respaldados por una placa metálica o por un laminado de fibras poliméricas en forma de panel mixto (Fig. 1), ofrecen una gran eficiencia merced a su acción erosiva sobre el proyectil $(1,2)$. Aunque en la producción de cerámicas estructurales avanzadas $\left(\mathrm{Al}_{2} \mathrm{O}_{3^{\prime}}, \mathrm{SiC}, \mathrm{NiAl}, \mathrm{TiB}_{2}\right)$ se ha evidenciado una progresiva reducción de sus costes de fabricación, la aplicación de estos materiales para la protección frente a impacto sigue estando restringida, por su elevado precio, a aplicaciones con estrictos condicionantes de ligereza $(3,4)$. En la Figura 2 se muestran las densidades superficiales de masa y los costes (relativos al de un acero convencional de blindajes) de diversas protecciones capaces de detener un proyectil $7.62 \mathrm{AP} \mathrm{a} 800 \mathrm{~m} / \mathrm{s}$. La eficiencia y el coste se ven sustancialmente incrementados cuando se emplea el material cerámico. El desarrollo de un material que pueda cubrir el salto existente entre los materiales metálicos y cerámicos puede ser de interés en aplicaciones en las que el peso no sea un factor de diseño determinante y se desee reducir el precio de la protección.

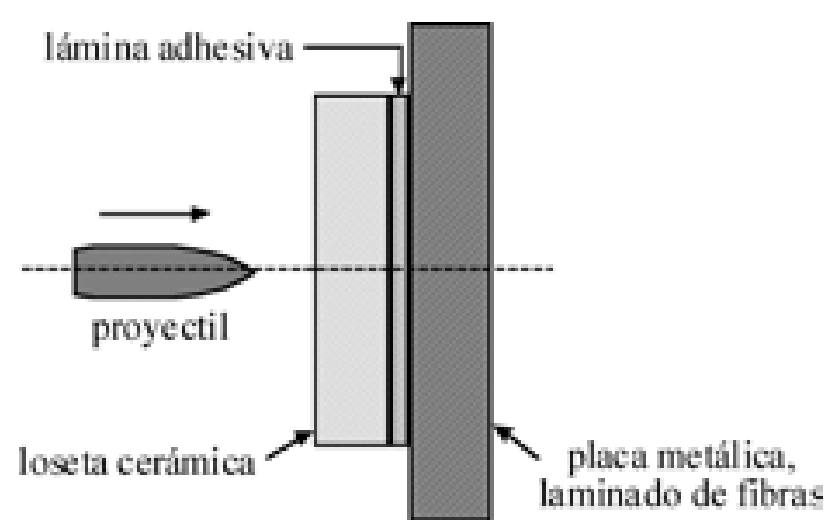

Figura 1- Protección ligera cerámica.

El objetivo de este trabajo ha consistido en el desarrollo de un material compuesto de matriz polimérica y carga cerámica que pueda ser empleado como alternativa a las cerámicas en los paneles mixtos. La labor se ha centrado esencialmente en la optimización del proceso de fabricación y en la caracterización mecánica del material en el rango de temperaturas a las que pueda verse sometido durante su servicio. 


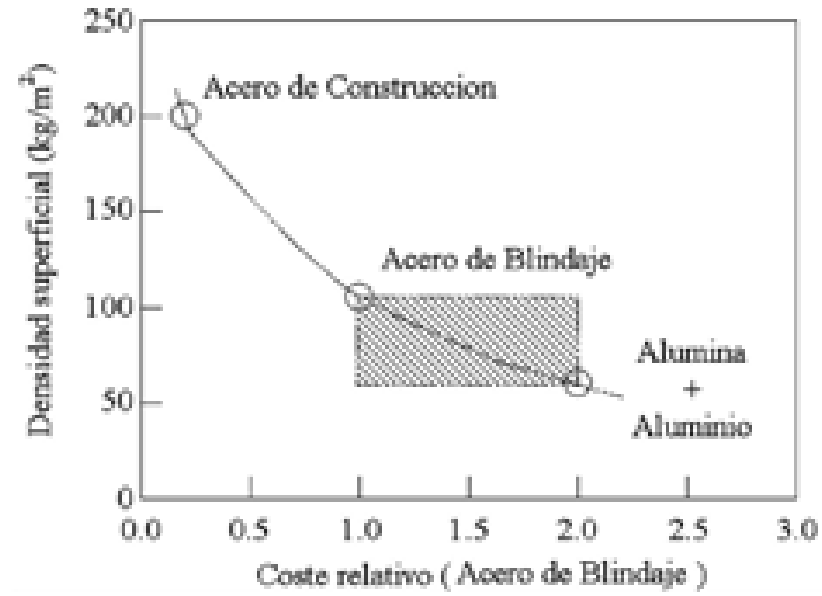

Figura 2.- Densidad superficial frente a coste (relativo al acero de blindaje) de diversas protecciones capaces de detener un proyectil $7.62 \mathrm{AP}$ a $800 \mathrm{~m} / \mathrm{s}(3)$

\section{MÉTODO DE FABRICACIÓN}

Como material para las partículas cerámicas se han elegido alúmina y carburo de silicio, por ser habitualmente empleados, en forma monolítica, en la protección ligera frente a impacto. De este modo, se seleccionaron partículas de $\mathrm{Al}_{2} \mathrm{O}_{3}$ (ALCOA T-60) de 99.4\% de pureza y partículas de SiC- $\alpha$ (NAVARROSIC RE) de $98.5 \%$ de pureza. Éstas se obtienen por electrofusión y son habitualmente empleadas como productos abrasivos y refractarios, por lo que presentan bajo coste y alta disponibilidad. El coste por unidad de masa de las partículas de alúmina es 30 veces inferior al correspondiente a la alúmina monolítica empleada habitualmente en la protección ligera frente a impacto. Como matriz polimérica se ha empleado la resina viniléster (EPOVIA RF-1001) por presentar buenas propiedades mecánicas y de trabajabilidad. En la Tabla I se muestran las propiedades mecánicas (módulo de elasticidad y límite elástico) de los materiales empleados, correspondientes a valores sumistrados por el fabricante y que fueron validadas en trabajos previos (5). Debido a que la misión de la cerámica es erosionar el proyectil y distribuir la carga en un área más amplia del panel posterior, el porcentaje de partículas debe ser lo más elevado posible aunque proporcionado con una cantidad de matriz que garantize la cohesión. Además, la porosidad del compuesto debe de ser baja para prevenir la prematura fragmentación y consiguiente deterioro del material durante el impacto (2).

TABla I. Módulo de ELASTICIDAd E Y LÍMITE ELÁSTICO Y DE LOS MATERIALES EMPLEADOS.

\begin{tabular}{|l|c|c|}
\hline & E [GPa] & Y [MPa] \\
\hline matriz vinilester & 2.5 & 90 \\
\hline partículas de $\mathrm{Al}_{2} \mathrm{O}_{3}$ & 290 & 2100 \\
\hline partículas de $\mathrm{SiC}$ & 350 & 2500 \\
\hline
\end{tabular}

El desarrollo de un método de fabricación de bajo coste ha permitido obtener un material compuesto con los requerimientos anteriores. Las etapas del proceso se resumen en: mezcla, compactación uniaxial, desmoldeo y postcurado. En la etapa de mezcla, las partículas se clasifican en diferentes husos granulométricos, al objeto de analizar la influencia del tamaño de grano en el comportamiento balístico de la protección, y posteriormente se combinan entre sí y con la resina en las proporciones deseadas. Es aconsejable que el tamaño del grano sea inferior a la mitad del espesor de la loseta para evitar heterogeneidades; en este trabajo se han empleado partículas con un tamaño máximo de $8 \mathrm{~mm}$. Por otra parte, el tamaño de las partículas tiene influencia en la porosidad del material compuesto. La Figura 3 muestra las densida-

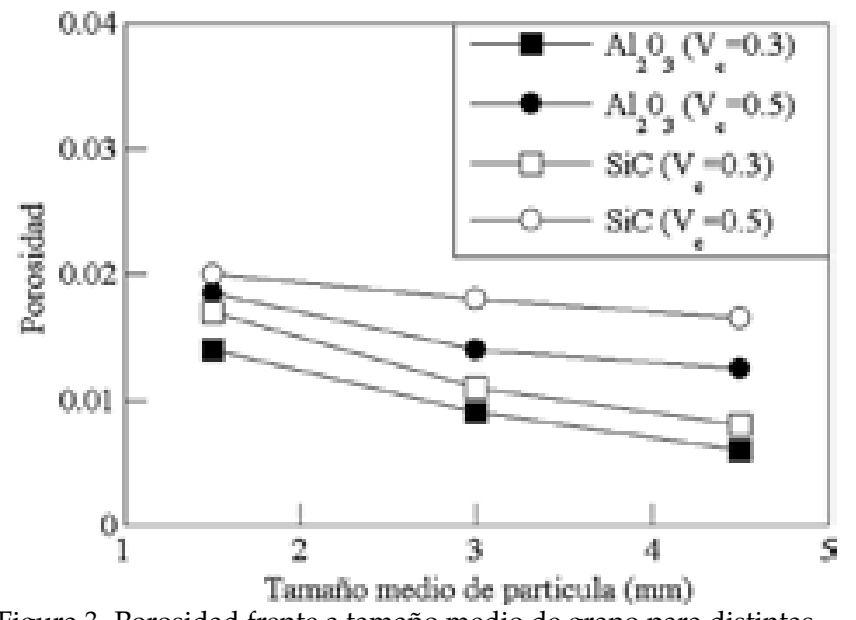

Figura 3.-Porosidad frente a tamaño medio de grano para distintas fracciones volumétricas de partículas $\mathrm{V}_{c}$.

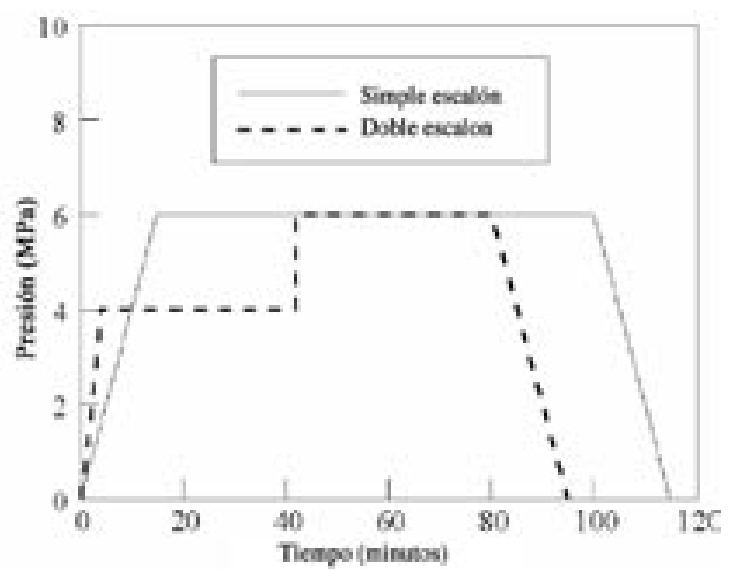

Figura 4.- Ciclos de compactación empleados en la fabricación del material compuesto.

des real y teórica de las losetas de material compuesto en función de la fracción volumétrica de partículas y del tamaño medio de grano. $\mathrm{Al}$ aumentar la fracción volumétrica de partículas y al disminuir el tamaño de grano, la porosidad aumenta debido al incremento de las superficies de contacto y del número de huecos. A mayor tamaño de partícula, la porosidad disminuye ya que las granulometrías de mayor tamaño dotan al compuesto de mayor compacidad (6).

El tiempo de polimerización y la trabajabilidad de la mezcla dependen de la proporción y orden de mezclado de los componentes: resina, partículas, catalizador (hidroperóxido de cumeno) y activador (octoato de cobalto). La compactación uniaxial ha sido necesaria para alcanzar un elevado contenido cerámico, ya que empaqueta las partículas, de acuerdo con experiencias previas en sinterización de materiales cerámicos (7), permite la evacuación de la resina y reduce la porosidad sustancialmente, haciendo innecesaria la aplicación de técnicas de vacío en el molde. Para la aplicación de presión, se ha empleado una máquina electromecánica Servosis de 100 kN de carga máxima haciendo uso de un utillaje específico. Las secuencias de compactación más usuales en sinterización de materiales cerámicos son las de simple escalón y doble escalón (8), recogidas en la Fig. 4. Analizadas las densidades de las probetas de material compuesto fabricadas empleando ambos métodos, se ha observado que la densidad es similar en ambos casos siempre que la velocidad de aplicación de la presión en el ciclo de simple escalón sea muy lenta. Por tanto, en la fabricación de las probetas, se ha utilizado el ciclo de simple escalón por su mayor sencillez de programación. 
El registro fuerza-desplazamiento del proceso de compactación ha permitido obtener la relación entre la presión aplicada, el porcentaje en peso de partículas y la densidad superficial de la loseta de material compuesto. La Fig. 5 muestra la relación entre la masa de partículas $m_{p^{\prime}}$ la masa final de la loseta $m_{f}$ y la presión aplicada. Cuanto mayor es la presión, mayor es la evacuación de la resina y mayor el porcentaje en peso de partículas que se obtiene en el material compuesto. A este respecto, fue necesaria la fabricación de utillaje de moldeo con la mínima holgura para permitir la evacuación de la resina sin pérdida de masa de partículas. De esta forma, se consigue reducir el contenido en resina, cuya única misión es aglomerar el material, manteniendo la misma capacidad erosiva con un menor peso global. Para presiones de $6 \mathrm{MPa}$ se han obtenido fracciones másicas de un $80 \%$ de material cerámico. Valores superiores de la presión producen fragmentación de las partículas cerámicas y pérdida de cohesión en el compuesto por excesiva evacuación de resina.

La Fig. 6 muestra la relación entre la densidad areal final de la loseta $\rho_{\mathrm{A}}$ la densidad inicial $\rho_{\mathrm{A} 0}$ y la presión de compactación (8). El proceso de compactación presenta dos fases. En la primera, hasta un valor aproximado de la presión igual a $3 \mathrm{MPa}$, las partículas se deslizan y se reordenan al aplicar la carga, produciéndose una mayor evacuación de resina y una mayor disminución de la densidad areal. Durante la segunda se produce una menor compactación ya que la resina comienza a polimerizar, por lo que la densificación es debida únicamente a la

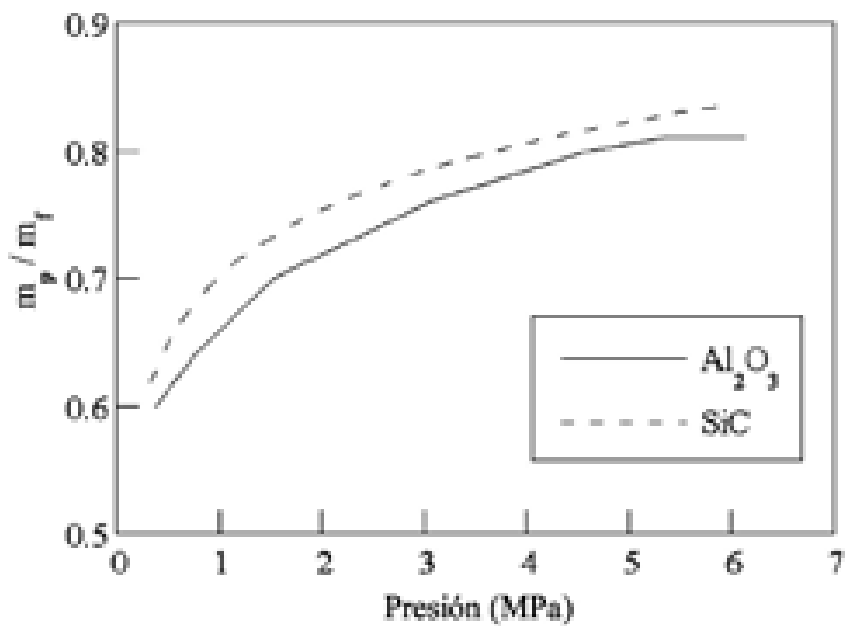

Figura 5.-Fracción másica de partículas $m_{p} / m_{f}$ frente a presión de compactación.

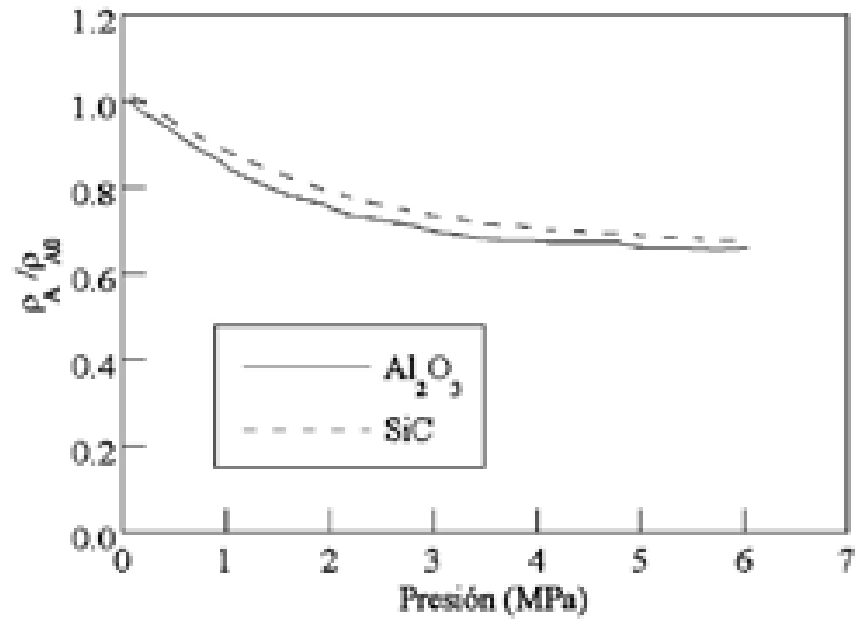

Figura 6.- Relación $\rho_{\mathrm{A}} / \rho_{\mathrm{A} 0}$ frente a presión de compactación. compresión volumétrica de la loseta. Después del desmoldeo el material compuesto fue curado en horno durante dos horas a $80^{\circ} \mathrm{C}$.

El proceso de fabricación desarrollado permite obtener con pocas operaciones losetas de material compuesto con un contenido en volumen de partículas cerámicas de hasta el $50 \%$ en volumen ( $80 \%$ en peso) y porosidad inferior al $2 \%$ a partir de materiales de bajo coste, controlando la densidad areal final de la loseta $\rho_{\mathrm{A}}$ y la proporción $m_{p} / m_{f}$.

\section{COMPORTAMIENTO ESTÁTICO A COMPRESIÓN}

Debido a que la eficiencia frente a impacto de las cerámicas está relacionada con su resistencia a compresión estática $(9,10)$, se ha analizado esta última propiedad para distintos tipos de material compuesto. Para ello se han realizado ensayos de compresión estática sobre probetas de $20 \times 20 \times 20 \mathrm{~mm}^{3}$ en una máquina universal Instron de 100 $\mathrm{kN}$, considerando varios factores: fracción volumétrica de partículas, tamaño medio de partícula, material de las partículas y temperatura de trabajo. Para cada combinación de factores se han ensayado 15 probetas.

La influencia de la fracción volumétrica de partículas se ha determinado considerando cuatro contenidos diferentes de carga cerámica (0, 30, 40 y 50\%). Las Figs. 7 y 8 muestran las curvas del comportamiento obtenidas a temperatura ambiente para distintas

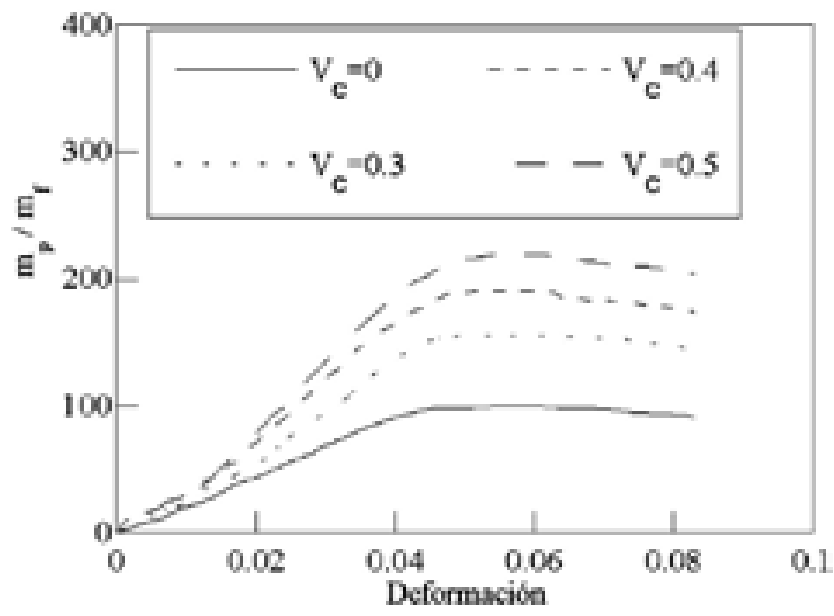

Figura.7.- Curvas estáticas tension-deformación del material compuesto para distintas fracciones volumétricas de $\mathrm{Al}_{2} \mathrm{O}_{3}$.

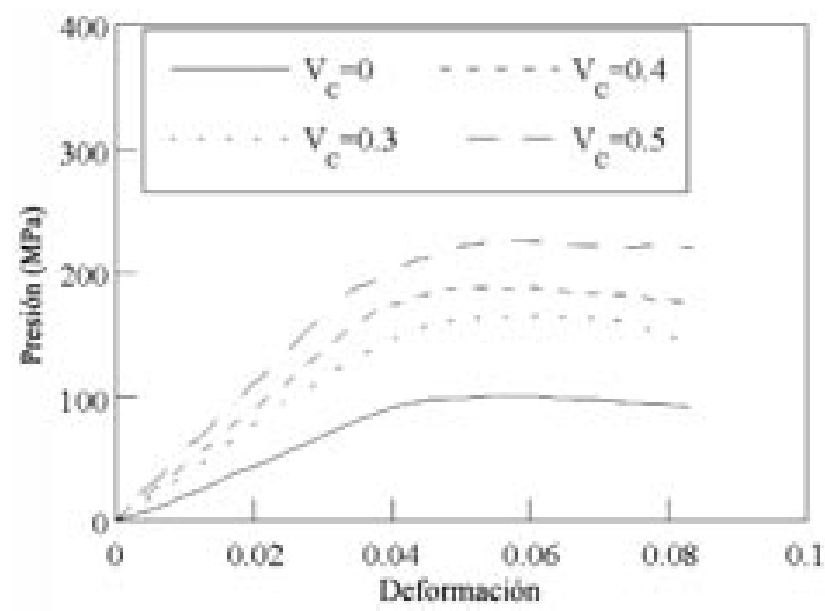

Figura.8.- Curvas estáticas tension-deformación del material compuesto para distintas fracciones volumétricas de $\mathrm{SiC}$. 
fracciones volumétricas de los dos materiales considerados: $\mathrm{Al}_{2} \mathrm{O}_{3}$ y $\mathrm{SiC}$, manteniendo constante el tamaño medio de partícula $(\delta=4.5$ $\mathrm{mm}$ ). El material compuesto presenta un comportamiento plástico que comienza en la zona convexa de la curva. Una vez alcanzada la tensión máxima se produce la fragmentación del material. En el rango elástico el comportamiento es no lineal hasta el 15\% del límite elástico a compresión y lineal a partir de este punto. Para los dos tipos de material cerámico empleado, las propiedades estáticas dependen fuertemente de la fracción volumétrica del refuerzo cerámico: una mayor cantidad de partículas incrementa el módulo de elasticidad y la tensión de plastificación. Esta tendencia, también apreciada por otros autores $(11,12)$ para otros materiales compuestos poliméricos (epoxi reforzado con micropartículas), es debida a que el principal mecanismo de deformación en probetas sometidas a compresión es producido por un campo de tensiones cortantes actuante sobre la matriz (13). Las partículas cerámicas, con propiedades resistentes muy superiores, resultan menos afectadas y contribuyen a rigidizar el compuesto. En cuanto a la influencia del material de las partículas, la comparación de las Figs. 7 y 8 permite observar, que aunque las partículas de $\mathrm{SiC}$ presentan valores más altos de módulo de elasticidad y límite elástico que las de alúmina (Tabla I), su incorporación a la matriz no se traduce de igual modo en una mejora de las propiedades del compuesto, ya que también es preciso considerar la adherencia partícula- matriz en este tipo de materiales (14).

Análogamente, los resultados experimentales muestran que el comportamiento resistente no se ve influido significativamente por el tamaño medio de partícula en el rango granulométrico considerado, tal como se aprecia en la Fig. 9.

Dado que las diferencias de temperatura ambiental pueden modificar el comportamiento de la matriz, se llevaron a cabo ensayos de compresión estática en el intervalo de temperaturas en que previsiblemente podría trabajar el material desarrollado, que se puede fijar entre $-15^{\circ} \mathrm{C}$ y $50{ }^{\circ} \mathrm{C}$. Las Figs. 10 y 11 muestran que la resistencia a compresión y el límite elástico disminuyen conforme aumenta la temperatura. Esta comportamiento, ya observado por otros autores (13) para materiales poliméricos reforzados con micropartículas cerámicas, es achacable al ablandamiento térmico de la resina polimérica, que sufre una pérdida de resistencia del $40 \%$ en el rango de temperaturas considerado, tal como se aprecia en ambas figuras.

\section{COMPORTAMIENTO FRENTE A IMPACTO DE ALTA VELOCIDAD}

El test de profundidad de penetración se emplea habitualmente para determinar la eficiencia frente a impacto de materiales cerámicos (15). En él, una loseta del material a evaluar se dispone sobre un bloque metálico de suficiente espesor y el conjunto es impactado por un proyectil. La penetración residual en el bloque metálico $p_{r}$ se compara entonces con la penetración de referencia $p$ en el mismo bloque sin loseta (Fig. 12) a través del factor de eficiencia másico FEM, propuesto por Hohler et al. (12), que se calcula mediante la expresión:

$$
F E M=\frac{\rho_{b} p}{\rho_{c} h_{c}+\rho_{b} p_{r}}
$$

donde $\rho_{b} y \rho_{c}$ son las densidades de metal y cerámica, respectivamente, y $h_{c}$ el espesor de la loseta cerámica. En este estudio se ha determinado el FEM para el aluminio 2017-T451, habitualmente empleado en protección contra impacto de alta velocidad, para alúmina monolítica de 95\% de pureza (Morgan Matroc AD 95 Sintox FA) y para el material compuesto desarrollado, tanto con carga de partículas de alúmina como de carburo de silicio. El proyectil utilizado ha sido un 7.62

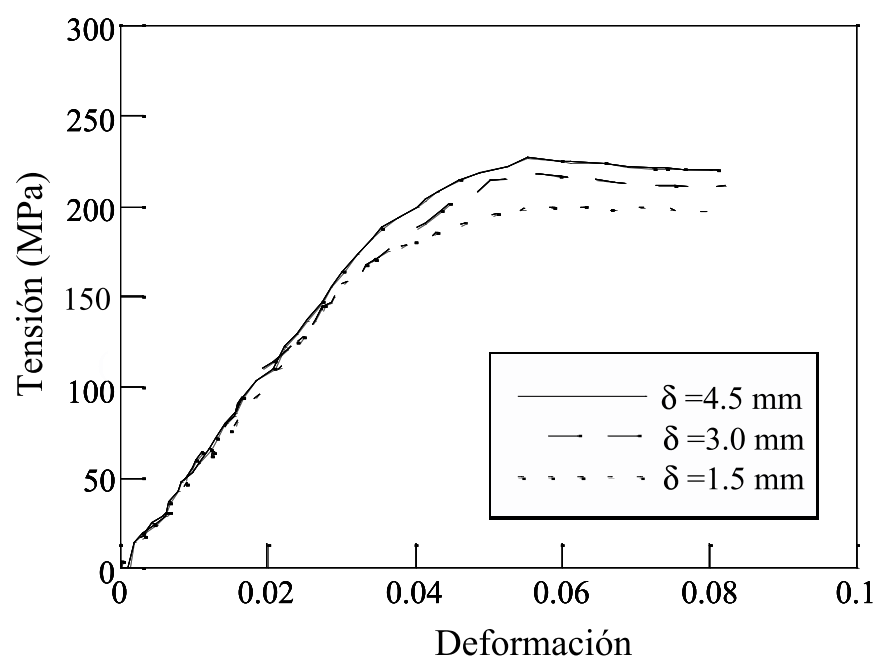

Figura. 9.- Curvas estáticas tensión-deformación del material compuesto con carga de partículas de $\mathrm{SiC}$ en función del tamaño medio de partícula.

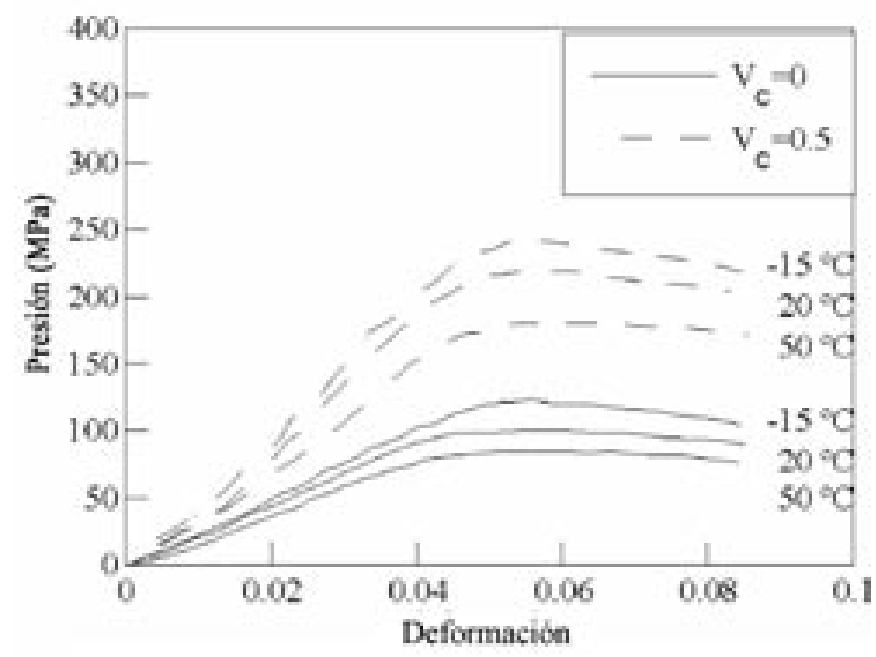

Figura.10.- Curvas estáticas tension-deformación del material compuesto con carga de partículas de $\mathrm{Al} 2 \mathrm{O} 3$ para distintas temperaturas de ensayo.

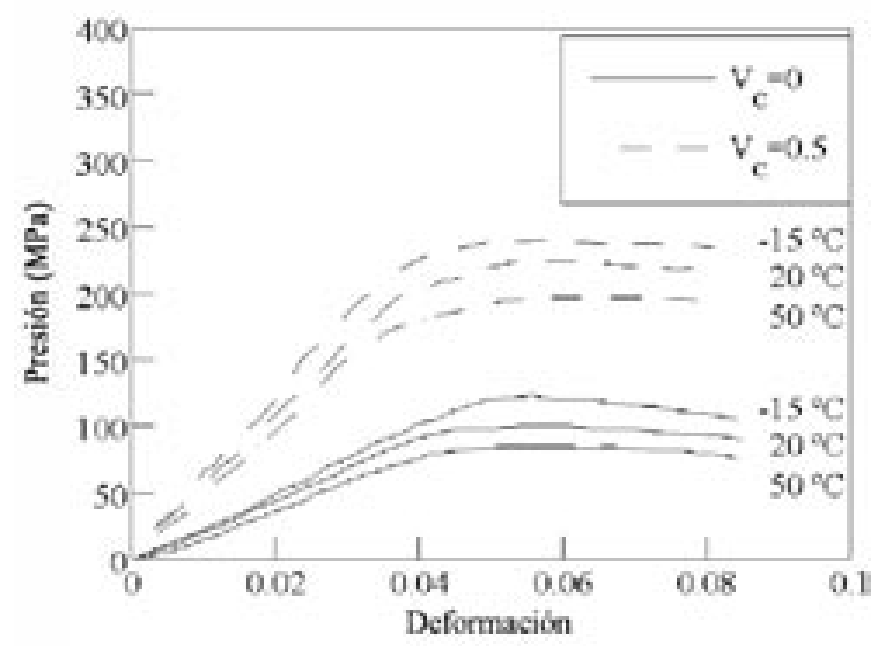

Figura.11.- Curvas estáticas tension-deformación del material compuesto con carga de partículas de $\mathrm{SiC}$ para distintas temperaturas de ensayo. 

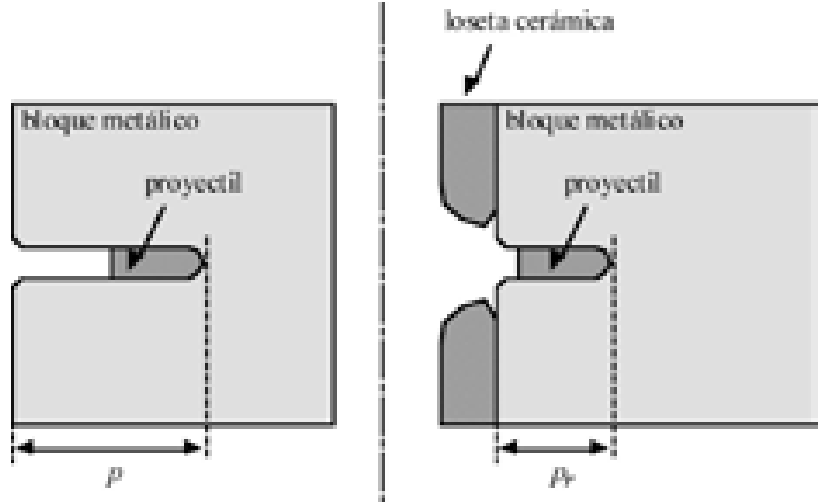

Fig. 12.- Configuración del test de Profundidad de Penetración.

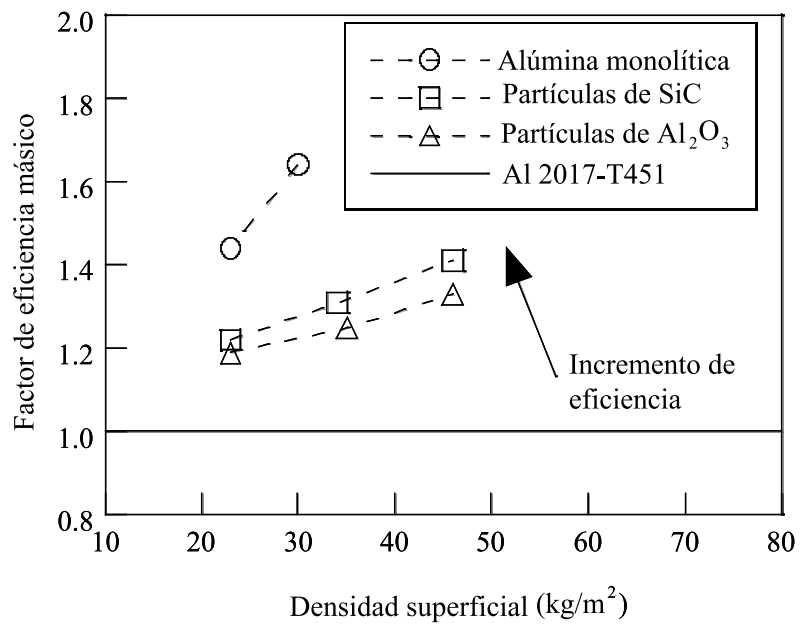

Fig. 13.- Factor de eficiencia másica frente a densidad superficial.

AP con núcleo de carburo de wolframio de $5.9 \mathrm{~g}$ de masa y relación longitud/diámetro igual a 3.6. La velocidad de impacto fue de 940 $\mathrm{m} / \mathrm{s}$. La penetración de referencia en el bloque metálico, de aluminio 2017-T451, fue de $42 \mathrm{~mm}$. Los resultados se muestran en la Fig. 13, donde se puede apreciar que la eficiencia del material compuesto es intermedia entre la del aluminio y la alúmina monolítica, constituyendo por lo tanto una alternativa a ambos materiales para aplicaciones en las que el peso no sea un condicionante de diseño determinante y se deseen reducir costes.

\section{CONCLUSIONES}

Se ha desarrollado un procedimiento de fabricación para el material compuesto de matriz polimérica y carga cerámica. Dentro de las diferentes fases del proceso, la compactación uniaxial ha resultado determinante para la obtención de losetas de material compuesto con un contenido en peso de partículas de hasta un $80 \%$ y una porosidad inferior al $2 \%$ sin recurrir a técnicas de vacío.

La resistencia a compresión cuasiestática del material compuesto se incrementa sensiblemente al aumentar la fracción volumétrica de partículas. El aumento del tamaño de grano produce así mismo una mejora de la resistencia, aunque su efecto no es tan significativo como el del anterior parámetro. El ablandamiento térmico de la matriz desaconseja su posible utilización a altas temperaturas.

Las losetas de material compuesto presentan una eficiencia frente a impacto intermedia entre la correspondiente al aluminio y a la de la cerámica monolítica, habitualmente empleados en este tipo de aplicaciones. La simplicidad del método de fabricación y el bajo precio de las materias primas permiten obtener un material compuesto de bajo coste. Todo ello justifica su aplicación en aquellos casos en los que el peso no sea un requisito excesivamente restrictivo.

\section{AGRADECIMIENTOS}

Los autores desean expresar su agradecimiento a la Comunidad Autónoma de Madrid por la financiación del Proyecto 07N / 0004 / 1998 que ha permitido la realización de este trabajo.

\section{BIBLIOGRAFÍA}

1. P.C. den Reijer. "Impact on ceramic faced armours". Ph. D. Thesis, Delft University of Technology (1991).

2. C. Navarro, R. Zaera, R Cortés, M. A. Martínez-Casanova. "The response of ceramic faced lightweight armours under projectile impact". Structures under Shock and Impact, III, 323-330 (1994).

3. C. J. Roberson. "Ceramic materials and their use in lightweight armour systems". Proceedings Lightweight Armour System Symposium. Royal Military Collegue of Science, Cranfield, England (1995).

4. B. Matchen. "Applications of ceramics in armor products". Key Engineering Materials 122 333-342 (1996).

5. A. Arias, R. Zaera, J. López-Puente, C. Navarro. “Numerical modeling of the impact behavior of new particulate-loaded composite materials". Composite Structures 61, 151-159 (2003).

6. P. Montoya-Jiménez, A.García-Meseguer, F. Morán-Cabré. "Hormigón armado", Editorial GG, Barcelona (1991).

7. G. Messing, C. Markhoff, L. McCoy "Characterization of ceramic powder compactation". Ceramic Bulletin 61: 8 857-860 (1982).

8. D. Bortzmeyer. "Dry pressing of ceramic powder" Ceramic Processing. Ed. Chapman and Hall (1995).

9. Z. Rozenberg , Y. Yeshurun. "The relation between ballistic efficiency and compressive strength of ceramic tiles". Int J. Impact Eng 7:3 357-362 (1988).

10. Z. Rozenberg, S J Bless, Y Yeshurun, K. Okajina "A new definition of ballistic efficiency based on the use of thick backing plates". In: Proccedings of Impact Conference, Bremen, (1987).

11. R J Young, P W Beaumont. "Effect of composition upon fracture of silica particle-filled epoxy resin composites". Journal Material Science 12:684-692 (1977).

12. O. Ishai, LJ Cohen. "Elastic properties of filled and porous epoxy composites". Int. J. Mech Science 9:539-46 (1967).

13. R. J. Young, D. Maxwell, A. Kinloch. Journal Material Science 21:380 (1986).

14. J.C. Smith. "Experimental values for the static constants and particulate-filled glassy polymer". J. Res NBS 80A:45-9 (1976).

15. V. Hohler, A J Stilp, K Weber. "Hypervelocity penetration of tungsten sintealloy rods into alumina". Int J. Impact Eng;17:409-418 (1995).

Recibido: 1.2.03

Aceptado: 30.11 .03 